\title{
On Imaging and Localizing Untethered Intraocular Devices with a Stationary Camera
}

\author{
Christos Bergeles, Kamran Shamaei, Jake J. Abbott, and Bradley J. Nelson
}

\begin{abstract}
Untethered microrobotic devices have been proposed for use in ophthalmic surgeries, and localization information is required for their control. The environment of the human eye is externally observable, and images can be used to localize intraocular agents. There is currently no quantitative description of the effects of the human eye optics on imaging and localizing intraocular objects. In this paper, the common methods of ophthalmoscopy assuming a stationary imaging system are examined and compared with respect to their imaging and localizing properties on a schematic model of the human eye. A mechatronic device that can image intraocular objects with increased field-of-view, spatial resolution, and depth sensitivity is proposed. 3D localization using a depth-from-focus approach is discussed, and the validity and limitations of a method based on paraxial approximations is demonstrated by experiments in a model eye.
\end{abstract}

\section{INTRODUCTION}

Vitreoretinal surgery requires accuracy and dexterity that is often beyond the limits of human surgeons. A number of robot-assisted solutions have been proposed and developed to improve surgeons' ability to perform these difficult procedures. Our work is motivated by the wireless magnetically steered intraocular microrobot proposed in [1]. For precise magnetic control of untethered devices, knowledge of the position of the device within the magnetic field is needed [2]. The interior of the human eye is externally observable, and thus, vision can be used to perform 3D localization.

Ophthalmic observation and surgery have been practiced for centuries, and clinicians have the ability to acquire high-definition and magnified images of the interior of the eye using an ever-increasing variety of optical tools that are designed specifically for the unique optical system that is the human eye. However, there is no prior work that explains the effects of the optics of the human eye on the localization of intraocular objects. The cornea, aqueous humor, pupil, intraocular lens, and vitreous humor (Fig. 1) limit the field-of-view, affect the formation of images (Fig. 2 ), and generally make localization challenging.

In order to avoid potential conflicts between the imaging system and the magnetic field steering system (or any other robot-assist system), we would ideally perform imaging with a stationary camera. Allowing motions of the optical system along its optical axis may be an acceptable exception. Common techniques for the localization of objects from monocular images involves the perspective projection of a 3D

This work was supported by the NCCR Co-Me of the Swiss National Science Foundation.

The authors are with the Institute of Robotics and Intelligent Systems, ETH Zurich, 8092 Zurich, Switzerland \{cbergeles, kamrans, jabbott, bnelson\}@ethz.ch

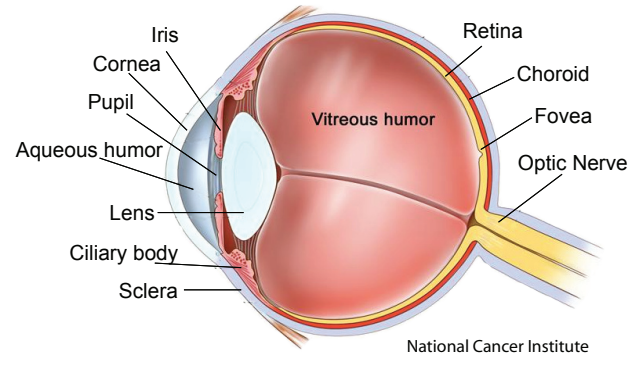

Fig. 1. Anatomy of the human eye.
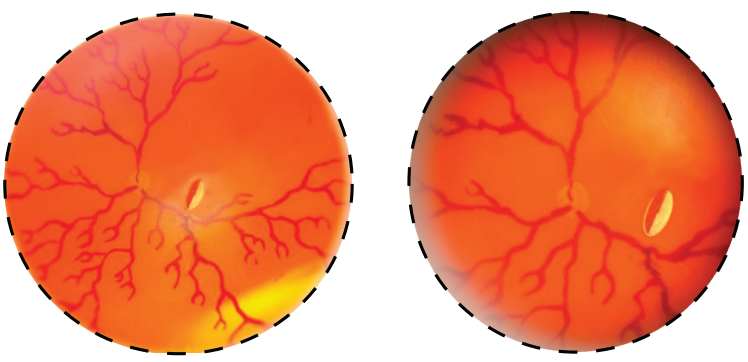

Fig. 2. The biomedical microrobot of [1] in a model eye [3]. The image on the left is the image of the intraocular environment without the optical elements, and the image on the right shows the effect of the model eye optics. Images are taken with a simple digital camera.

CAD model onto a 2D image plane [4], assuming a calibrated imaging system. However, in our case, calibrating the entire optical system is infeasible because of the inaccesibility of the interior of the human eye, as well as its unique optics.

Other methods for depth extraction involve using focus/defocus information [5], [6]. These methods do not require a model of the object, but only knowledge of the optical system. Depth-from-focus methods extract depth information by bringing an image into focus, whereas depthfrom-defocus methods rely on the amount of defocusing in an image to estimate depth. In [7], [8], methods to track fluorescent particles in fluids using defocus information are presented. Were depth-from-focus/defocus methods to be applied in the human eye, they could be used to localize not only modeled devices, but also unknown objects such as floaters or foreign bodies. Depth from defocus is proposed in [9] as a method to visually servo intraocular microrobots, however, the optics of the eye are not properly considered.

In this paper, we account for the optics of the human eye in the imaging and localization of intraocular objects with a stationary camera. We begin by comparing different ophthalmoscopy methods with regard to imaging and 
localization. Based on our findings, we propose a novel system that captures a wide field-of-view of the eye and can be used to localize intraocular objects by extracting depth information from focus. We show that if biometric measurements of the human eye are available, accurate localization can be achieved. Finally, we demonstrate the applicability and limitations of a localization method using a first-order approximation through experiments in a model eye. We will consider only the relaxed (or paralyzed) lens of an emmetropic eye, which focuses parallel incoming rays on the retina. Consequently, although there may be variations in the optical systems between patients, we consider a given patient's eye as a static optical system. We will also assume that we have sufficient illumination to acquire our images.

\section{Comparison OF Ophthalmoscopy Methods}

We begin by investigating the feasibility of imaging and localizing intraocular devices using existing ophthalmoscopy methods. The first approach involves looking directly into the human eye, and is analogous to direct ophthalmoscopy. The second approach involves the utilization of vitrectomy contact lenses. Finally, we consider the use of a non-contact condensing lens between the eye and the imaging system. This method is analogous to indirect ophthalmoscopy.

Our results are based on Navarro's schematic eye [10]. Schematic eyes are models that explain the optical properties of the human eye, and are based on biometric data from population samples. Navarro's schematic eye performs well for angles up to $\sim 70^{\circ}$, measured from the center of the pupil and around the optical axis. Simulations are carried out with the OSLO optical lens design software. Throughout this section, the object's depth $z$ is measured along the optical axis.

\section{A. Direct Ophthalmoscopy}

In a relaxed state, the retina is projected through the eye optics as a virtual image at infinity. The parallel beams can be captured by an imaging system, and the image of the retina is created. In direct ophthalmoscopy - the simplest method of intraocular observation-the rays are brought in focus on the observer's retina [12]. Using the formulas of [13], and after some manipulation, we can calculate the field-of-view for direct ophthalmoscopy at $\sim 10^{\circ}$ (Fig. 3(a)).

Every object inside the eye will create a virtual image. These virtual images approach infinity rapidly as the object approaches the retina. Fig. 3(c) displays the distance where the virtual image is formed versus different positions of an intraocular object. In order to capture the virtual images that are created from objects close to the retina, an imaging system with close to infinite working distance is required. Such an imaging system will also have a large depthof-field. As a result, depth information from focus would be insensitive to object position. Imaging with an optical microscope would be impossible due to its limited working distance.

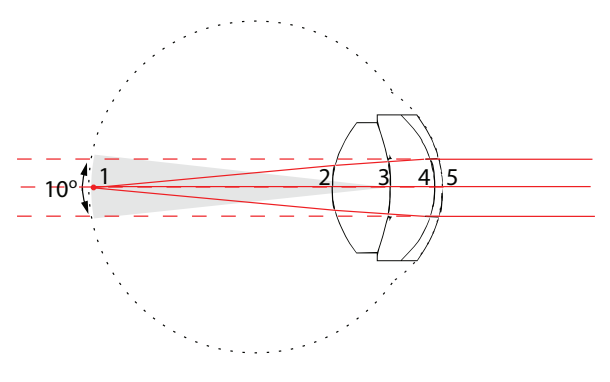

(a)

$\begin{array}{cr}\text { Surface } & \text { Radius } \\ 1 & 12.00 \mathrm{~mm} \\ 2 & 6.00 \mathrm{~mm} \\ 3 & -10.20 \mathrm{~mm} \\ 4 & -6.50 \mathrm{~mm} \\ 5 & -7.72 \mathrm{~mm}\end{array}$

Conic Constant Thickness

$\begin{array}{rr}0.00 & 16.32 \mathrm{~mm} \\ -1.00 & 4.00 \mathrm{~mm}\end{array}$

$-3.13 \quad 3.05 \mathrm{~mm}$

$0.00 \quad 0.55 \mathrm{~mm}$

(b)

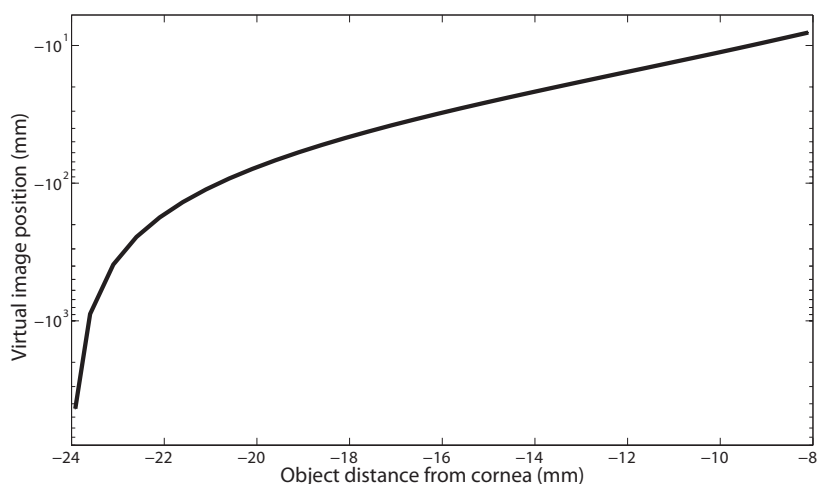

(c)

Fig. 3. (a) Navarro's schematic eye (b) Optical parameters (c) Virtual image position versus intraocular object position for the direct ophthalmoscopy case (image distances are measured from surface 5).

\section{B. Vitrectomy Lenses}

To visualize objects in the vitreous humor of patients, a variety of contact lenses can be used. For robotic agents that operate in the vitreous humor of phakic (i.e. intact intraocular lens) eyes, only the use of plano-concave lenses (Fig. 4(a)) needs to be considered [12].

Vitrectomy lenses cause the virtual images of intraocular objects to form inside the eye, allowing the imaging systems to have a reduced working distance. Based on data given from HUCO Vision SA for the vitrectomy lens S5.7010 [11], we simulated the effects of a plano-concave vitrectomy lens on Navarro's eye (Fig. 4(a)). This vitrectomy lens allows a field-of-view of $\sim 40^{\circ}$, significantly increased from the direct observation of Sec. II-A.

As shown in Fig. 4(c), the virtual images are formed inside the eye and span a lesser distance. Thus, contrary to direct observation, imaging with an optical microscope (relatively short working distance and depth-of-field) is possible. The working distance of such an imaging system must be at least $20 \mathrm{~mm}$. As depth-of-field is proportional to working distance, there is a fundamental limit to depth-from-focus resolution acheivable with vitrectomy lenses. 


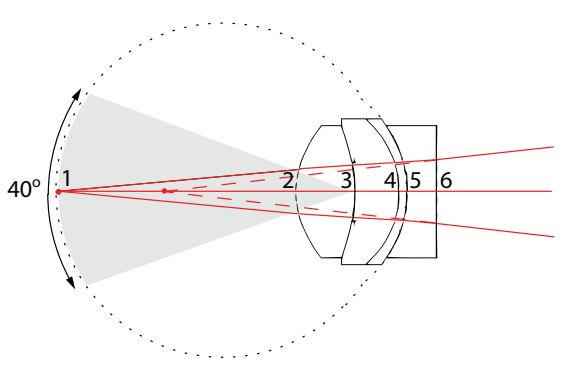

(a)

$\begin{array}{crrcc}\text { Surface } & \text { Radius } & \text { Conic Constant } & \text { Thickness } & \text { Refraction Index } \\ 1 & 12.00 \mathrm{~mm} & 0.00 & 16.32 \mathrm{~mm} & 1.336 \\ 2 & 6.00 \mathrm{~mm} & -1.00 & 4.00 \mathrm{~mm} & 1.420 \\ 3 & -10.20 \mathrm{~mm} & -3.13 & 3.05 \mathrm{~mm} & 1.337 \\ 4 & -6.50 \mathrm{~mm} & 0.00 & 0.55 \mathrm{~mm} & 1.376 \\ 5 & -7.72 \mathrm{~mm} & -0.26 & 2.00 \mathrm{~mm} & 1.425 \\ 6 & \infty & 0.00 & \infty & 1.000\end{array}$

(b)

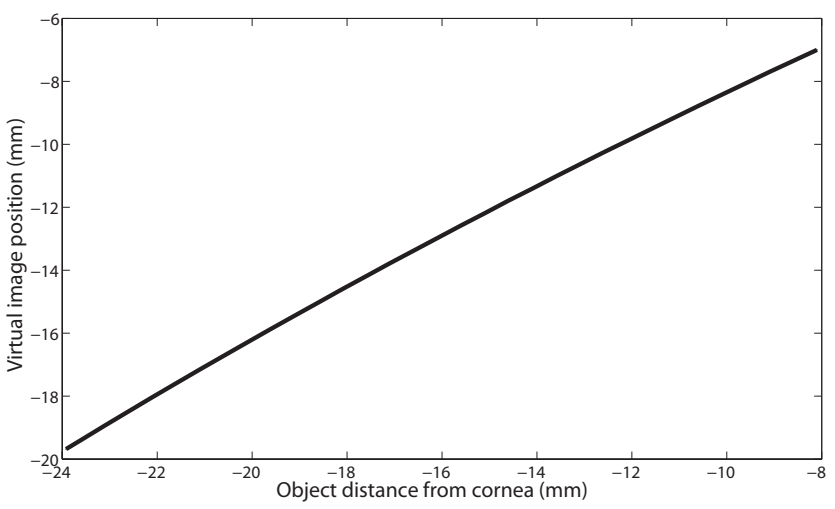

(c)

Fig. 4. (a) Navarro's schematic eye with a vitrectomy lens [11] (b) Optical parameters (c) Virtual image position versus intraocular object position for the vitrectomy case (image distances are measured surface 6).

\section{Indirect Ophthalmoscopy}

Indirect ophthalmoscopy (Fig. 5(a)) allows a wider field of the retina to be observed. A condensing lens is placed in front of the patient's eye, and catches rays emanating from his peripheral retina. These rays are focused after the condensing lens, creating an aerial image of the patient's retina. The condensing lenses compensate for the optical aberrations of the human eye and its refractive effects, creating focused images of the entire retinal fundus.

We simulated the effects of a double aspheric condensing lens based on information found in [14]. This lens, when placed $\sim 5 \mathrm{~mm}$ from the pupil, allows imaging of the peripheral retina and offers a field-of-view of $\sim 100^{\circ}$. As a result, it can be part of an imaging system with superior field-of-view than the ones described in Sec. II-A and Sec. IIB. The image positions versus the intraocular object positions can be seen in Fig. 5(c). A sensing system with a short working distance and shallow depth-of-field can be used in order to extract depth information from focus, for all areas inside the human eye. Depth estimation is more sensitive for objects near the intraocular lens, since smaller object

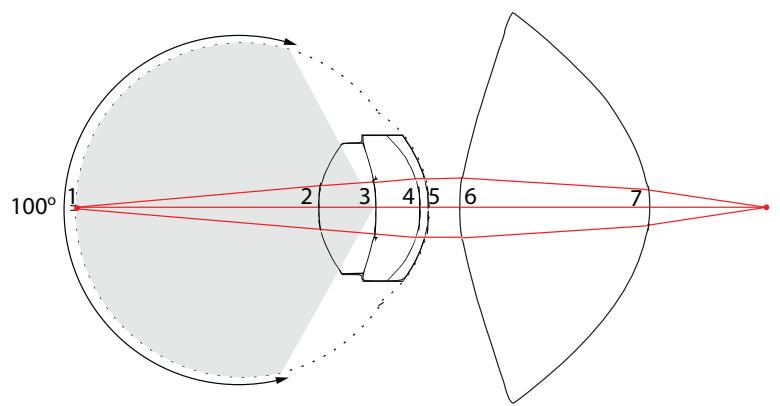

(a)

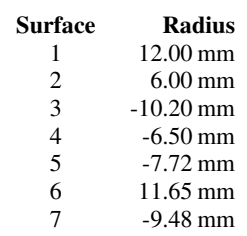

Conic Constant Thickness

$\begin{array}{rrc}\text { Thickness } & \text { Refraction Inde } \\ 0.00 & 16.32 \mathrm{~mm} & 1.336 \\ -1.00 & 4.00 \mathrm{~mm} & 1.420 \\ -3.13 & 3.05 \mathrm{~mm} & 1.337 \\ 0.00 & 0.55 \mathrm{~mm} & 1.376 \\ -0.26 & 2.31 \mathrm{~mm} & 1.000 \\ -9.24 & 13.00 \mathrm{~mm} & 1.523 \\ -1.07 & \infty & 1.000\end{array}$

(b)

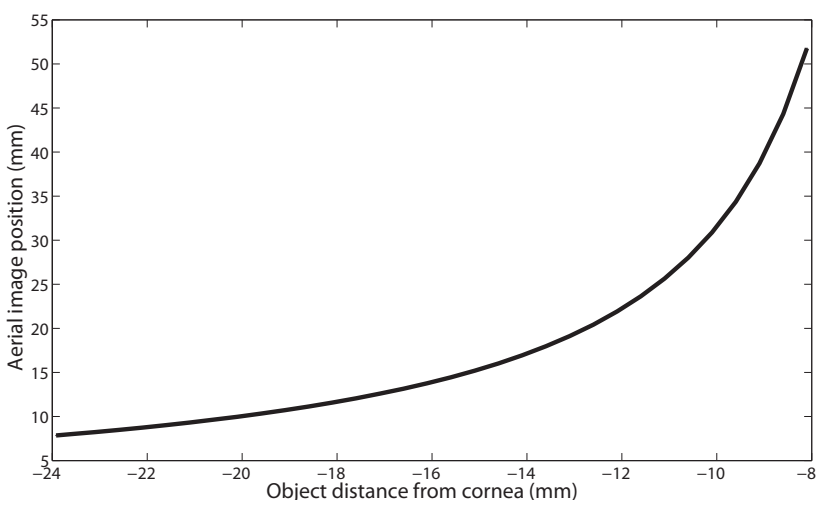

(c)

Fig. 5. (a) Navarro's schematic eye with a condensing lens [14] (b) Optical parameters (c) Virtual image position versus intraocular object position for the indirect ophthalmoscopy case (image distances are measured from surface 7 ).

displacements result in larger required focusing motions.

\section{A Mechatronic Ophthalmoscope For Imaging AND LOCALIZING INTRAOCULAR DEVICES}

Inspired from the findings of Sec. II, we created a mechatronic vitreoretinal ophthalmoscope (MVO) (Fig. 6) based on indirect ophthalmoscopy. The MVO is a simple device that consists of two components: a condensing lens that is kept at a constant position with respect to the eye, and a sensor that captures the aerial image directly and moves with respect to the lens to focus on objects throughout the eye. The Digital Wide Field ${ }^{\circledR}$ [15] lens from Volk Optical Inc. is used as the condensing lens. A $24 \times 24 \mathrm{~mm}^{2}$ CMOS sensor can be used to capture the full field-of-view.

The condensing lens causes a magnification of $0.72 \times$ and thus, a structure of $100 \mu \mathrm{m}$ on or near the retina will create an image of $72 \mu \mathrm{m}$. Even with no additional magnification, a CMOS sensor with a common sensing element size of $6 \times 6 \mu \mathrm{m}^{2}$ will be able to resolve small retinal structures sufficiently. Dense CMOS sensors have a shallow depthof-focus, and as a result, they can be used effectively in 


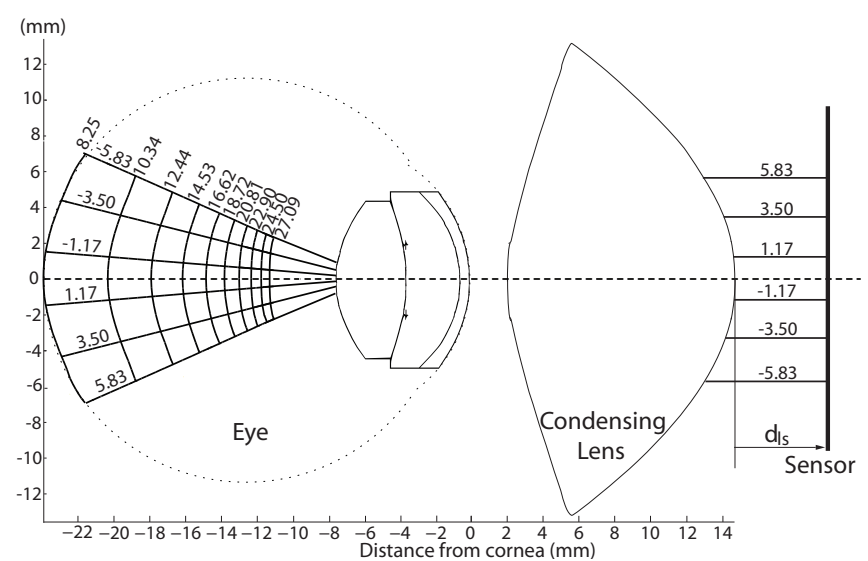

Fig. 6. Simulation of the mechatronic vitreoretinal ophthalmoscope, with the isofocus surfaces and isopixel curves, for the system of Fig. 5(a). The different isofocus surfaces correspond to values of the sensor position $d_{l s}$ in $\mathrm{mm}$, for uniform sensor steps of $\sim 2.1 \mathrm{~mm}$. The isopixel curves correspond to pixel distances from the optical axis for uniform steps of $\sim 2.3 \mathrm{~mm}$.

depth-from-focus techniques. Based on Fig. 5(c), to focus on objects throughout the eye, the sensor must have a travel of $\sim 45 \mathrm{~mm}$. However, since we are interested in localizing objects mainly in the posterior of the eye, a sensor travel of only $\sim 10 \mathrm{~mm}$ is necessary.

An additional benefit of the MVO is that it minimizes the amount of illumination necessary for adequate image quality. Since the human eye, especially the retina, is a sensitive structure, any method that minimizes irradiance on it is preferable. Every lens surface attenuates the light passing through. Thus, for every additional surface we must exert an extra amount of light on the eye. The MVO allows the minimum light intensity to be exerted on the patient's eye by minimizing the number of lens surfaces within the light path to two, whereas the methods of Sec. II-A and Sec. II-B will require more lens surfaces to capture the virtual images. This minimal requirement for retina irradiance, in combination with the wide field-of-view, adequate spatial resolution, and ability to obtain depth information from focus, makes the MVO promising for imaging and localizing intraocular devices.

As previously stated, the condensing lens projects the spherical surface of the retina on a flat aerial image. One expects that moving the sensor will focus the image at different surfaces inside the eye; we call these surfaces isofocus surfaces. Moreover, locations inside the eye will correspond to pixels on a moving sensor in a way that differs from the perspective projection model; the locus of intraocular points that is imaged on the same pixel coordinates is called an isopixel curve.

We estimate the isofocus surfaces and the isopixel curves with exact raytracing. Due to the rotational symmetry of the system, we examine the 2D case. For a grid of points inside Navarro's eye, a fan of rays is traced until the sensor position. We position the sensor plane so that the spot size created by this rayfan is minimized (i.e. the image is in focus). The 2D coordinates on the sensor plane where the rayfan is focused specify the pixel coordinates on the image. With the calculated information we create the isofocus surfaces and isopixel curves. The results within the area of validity of the Navarro eye can be seen in Fig. 6. The position of an intraocular point can be estimated from the intersection of its isopixel curve with its isofocus surface determined from $d_{l s}$.

Fig. 6 shows that the expected depth resolution is higher for regions far from the retina. Moreover, it shows that the formed image is inverted. From the slope of the isopixel curves, it is understood that the magnification of the intraocular object increases farther from the retina. As a result, we conclude that both spatial and lateral resolution increase for positions closer to the intraocular lens. Furthermore, as the intraocular lens is approached, the isofocus surfaces transition from spherical surfaces to planes.

\section{Localization Using a Paraxial Model}

The first and simplest method to consider for intraocular localization uses a paraxial model. Paraxial models are firstorder models of the optics of a system of lenses, which hold in regions near the optical axis. The accuracy of these methods decreases as the angles of the incoming rays with respect to the optical axis increase, and the breakdown point of the approximations depends on the lenses that compose the combined optical system.

We conducted a depth-from-focus experiment on an aligned imaging system (Fig. 7) that consists of the MVO and a model eye [3], in order to extract the precise relation between the intraocular object and the in-focus sensor position. A circular calibration pattern was moved with $1 \mathrm{~mm}$ steps in the model eye. The image sensor and object were translated using Sutter stages. The required focus scores were calculated with techniques described in [16]. In order to extract a paraxial model of the condensing lens (which is symmetric around the optical axis), we fit first-order curves on its sides in an image (Fig. 8). With thick lens equations [17] we can calculate the equivalent focal length and the principal planes for the condensing lens:

$$
\begin{aligned}
f_{l}^{-1} & =\left(n_{l}-1\right)\left(\frac{1}{R_{1}}-\frac{1}{R_{2}}+\frac{n_{l}-1}{n_{l} R_{1} R_{2}} d_{l}\right) \\
{\left[O_{2}, H_{1}\right] } & =\frac{-f_{l}\left(n_{l}-1\right) d_{l}}{R_{2} n_{l}} \\
{\left[O_{3}, H_{2}\right] } & =\frac{-f_{l}\left(n_{l}-1\right) d_{l}}{R_{1} n_{l}}
\end{aligned}
$$

and for the compound imaging system:

$$
\begin{aligned}
f_{c}^{-1} & =\frac{1}{f_{e}}+\frac{1}{f_{l}}-\frac{d_{e l}+\left[O_{2}, H_{1}\right]}{f_{e} f_{l}} \\
{\left[O_{1}, H_{c 1}\right] } & =\frac{f_{c}\left(d_{e l}+\left[O_{2}, H_{1}\right]\right)}{f_{l}} \\
{\left[H_{2}, H_{c 2}\right] } & =\frac{f_{c}\left(d_{e l}+\left[O_{2}, H_{1}\right]\right)}{f_{e}}
\end{aligned}
$$

where $d_{o e}=31.0 \mathrm{~mm}, f_{e}=30.00 \mathrm{~mm}, R_{1}=21.71 \mathrm{~mm}$ and $R_{2}=-11.08 \mathrm{~mm}$ (the radii of surfaces 1 and 2 of Fig. 7, respectively) were measured, $d_{e l}=6.22 \mathrm{~mm}$ was empirically chosen based on preliminary experiments, and $n_{l}=1.65$ is 


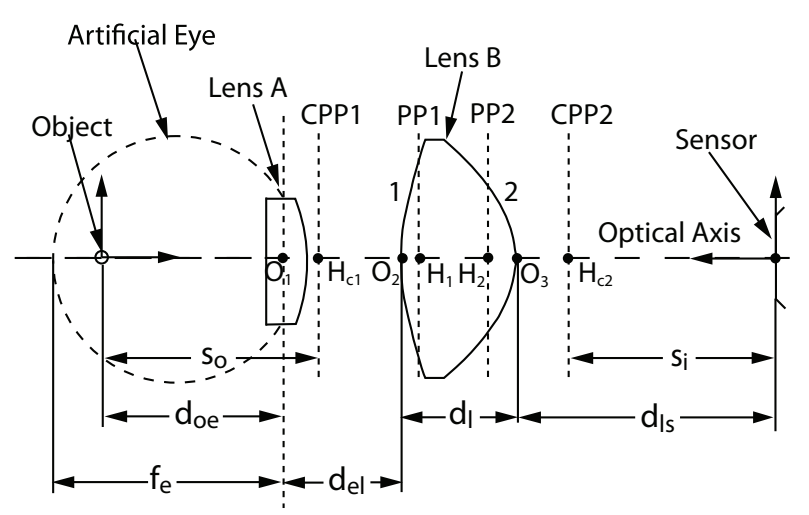

Fig. 7. Experimental imaging setup. PP: principal planes of the thick condensing lens, CPP: principal planes of the compound lens system.

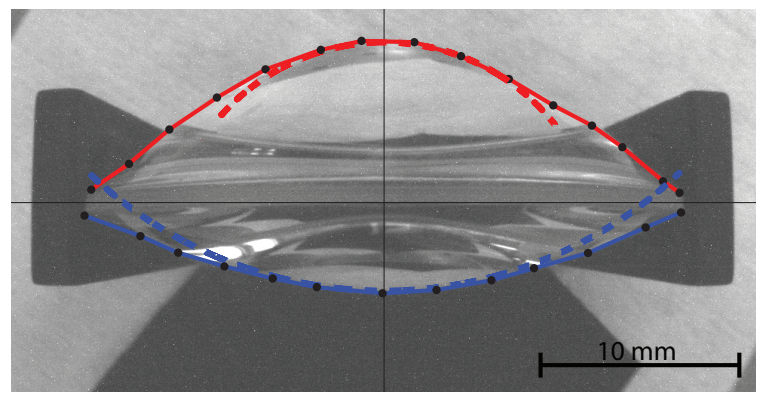

Fig. 8. The solid curves correspond to the aspheric models of the lens, and the dashed curves correspond to their paraxial approximations. The blue (lower) curves corresponds to the surface 1 in Fig. 7, and the red (upper) curves to surface 2 . The black dots correspond to the points chosen for the model fits.

an estimated refractive index of the condensing lens based on [14] (see Fig. 7 for the explanation of each parameter). The operator $[\cdot, \cdot]$ denotes the signed distance between two points.

The compound system projects an object at $s_{o}$ to an image on the sensor at distance $s_{i}$ :

$$
\begin{aligned}
s_{i} & =d_{l s}-\left[O_{3}, H_{2}\right]-\left[H_{2}, H_{c 2}\right]+e_{s_{i}} \\
s_{o} & =\frac{f_{c} s_{i}}{s_{i}-f_{c}} \\
d_{o e} & =s_{o}-\left[O_{1}, H_{c 1}\right]+e_{d_{o e}}
\end{aligned}
$$

where $d_{l s}$ is our control variable. Due to measurement and calculation uncertainties, there are accumulated errors that can be lumped and included as errors in the estimated image $\left(e_{s_{i}}\right)$ and object $\left(e_{d_{o e}}\right)$ positions.

In the model eye it is possible to calibrate for the errors using the actual values, and the result is shown in Fig. 9. In reality, limited access to the interior of the human eye makes full calibration impossible. However, from biometric measurements, the position of the retina can be known. Assuming that we have knowledge of the true retinal position, we can calibrate our system and estimate the errors $e_{s_{i}}$ and $e_{d_{o e}}$ from only one initial observation. In Fig. 9 the biometric model is fit on the actual values using just one point for calibration. Using this method, the accuracy will always be high near the retina, but has the potential to decrease for

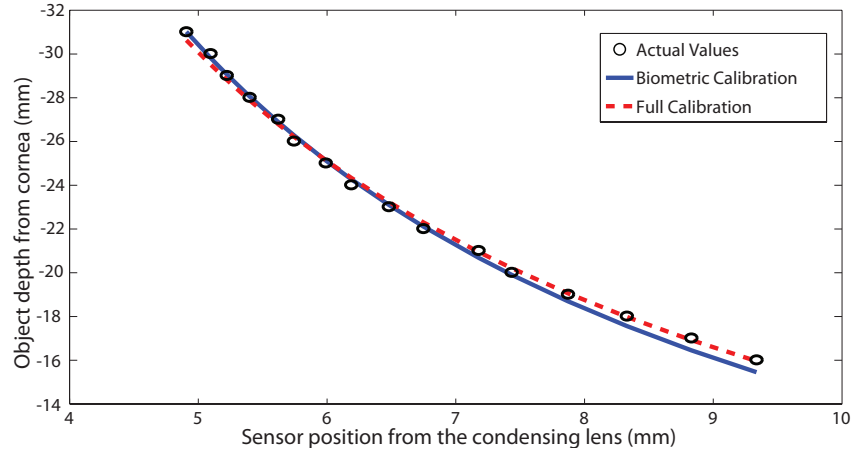

Fig. 9. Actual values and model fits for the depth-from-focus experiment. Full calibration shows a mean absolute error of $190 \mu \mathrm{m}$. Biometric calibration shows a mean absolute error of $224 \mu \mathrm{m}$.

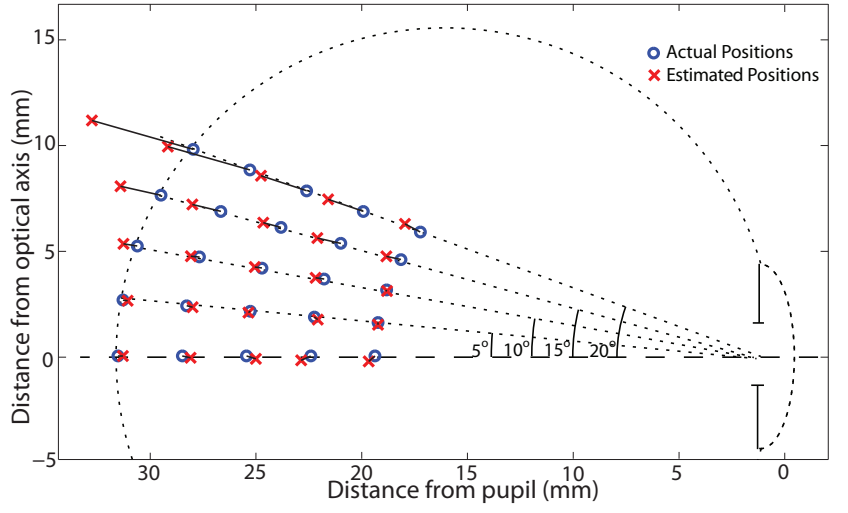

Fig. 10. Localization experiment showing the limits of paraxial approximations. Experiment is shown for the model eye [3].

regions closer to the pupil.

In order to perform full 3D localization after estimating the intraocular object depth, we take advantage of the concept of the nodal points of a lens system: a ray reaching the first nodal point will exit the system from the second nodal point with the same angle. The lens system can be eliminated, and can be considered as a pinhole camera located at the first nodal point. The focal length of the pinhole camera is $s_{i}$ calculated from (7). As a result, we can estimate the 3D coordinates of an intraocular point by knowing its pixel coordinates and the sensor position:

$$
\begin{aligned}
& z_{\text {est }}=s_{o} \\
& x_{\text {est }}=k_{x}\left(u-u_{o}\right) \frac{z_{\text {est }}}{s_{i}} \\
& y_{\text {est }}=k_{y}\left(v-v_{o}\right) \frac{z_{\text {est }}}{s_{i}}
\end{aligned}
$$

where $s_{o}$ is given in (8), $(u, v)$ and $\left(u_{o}, v_{o}\right)$ are the 2D pixel coordinates of the intraocular point and the center of the image, respectively, and $k_{x}, k_{y}$ are the dimensions of the pixel elements.

In order to estimate the area of validity of the paraxial approximation, we consider points on the retina of the model eye, and we perform a localization experiment for various angles with respect to the optical axis, and decreasing distances from the pupil. The results are shown in Fig. 
10. Localization errors are minimal for angles smaller than $\sim 10^{\circ}$ from the optical axis.

We can identify two main reasons responsible for the failure of the paraxial method at a wider field-of-view. Firstly, the first-order models involve small-angle assumptions. Secondly, the condensing lens is projecting a spherical surface on a plane, and paraxial approximations assume a plane-toplane mapping for the estimation of depth. In Fig. 6 it was shown that the isofocus surfaces transition to planes closer to the lens. This explains the error's decrease in Fig. 10 as the object approaches the pupil.

\section{Summary AND Discussion}

In this paper, the basic approaches to image and localize intraocular devices have been presented. The selection of the imaging system must consider the virtual/aerial images that are created due to the optics of the eye and any additional lenses. A mechatronic vitreoretinal ophthalmoscope with advantages in field-of-view, spatial resolution, and illumination has been proposed, and its abilities to localize intraocular objects have been discussed. The commonly used paraxial optical model was shown to be accurate for small angles, but erroneous for wide-field experiments.

To take advantage of the full field-of-view of the MVO, we must go beyond the simple paraxial model. In Fig. 6 it was shown that localization can be performed by finding the isofocus surfaces and isopixel curves of the combined optical system. These surfaces and curves can be computed offline if each of the optical elements in the system is well modeled. It is possible to acquire higher-order models for the surfaces of the condensing lens, as shown in Fig. 8. For the human eye optics, it may be possible to use a schematic eye, which represents a mean population model. However, if this results in unacceptable localization errors, biometric measurements can be taken on individual patients, using techniques such as those described in [18], [19].

The isopixel curves are lines, and it is straightforward to parametrize them using their slope and their point of intersection with the optical axis. Each isopixel curve corresponds to one pixel on the image, and its two parameters are functions of the corresponding pixel's radius (measured from the image center) due to the rotational symmetry of the system. However, these functions are yet to be determined. The isofocus surfaces result from the optics of a rotationally symmetric, aligned system composed of conic surfaces. We can thus assume that they are also conic surfaces that can be parametrized by their conic constant, radius of curvature, and intersection with the optical axis. Since the isofocus surfaces correspond to a specific sensor position, their three parameters can also be expressed as functions of the sensor position. However, these functions are also yet to be determined.

To fully control untethered devices, their position and orientation (i.e. their pose) must be estimated. In this paper we addressed position estimation. For the estimation of orientation, methods exist for perspective projection systems [4]. These methods work by projecting an estimate of the 6-DOF pose of a CAD model of the device on the image, based on a calibrated camera projection matrix, and then adapting the estimate of the pose until projection agrees with the perceived image. It may be possible to apply similar methods for pose estimation of intraocular objects. However, the estimates of the pose would be projected on the image by making use of the isopixel curves. This topic is left as future work.

\section{ACKNOWLEDGMENT}

The authors would like to thank Ioannis Kaliakatsos from the Institute of Robotics and Intelligent Systems, ETH Zurich, for his help with the construction of the experimental setup.

\section{REFERENCES}

[1] K. B. Yesin, K. Vollmers, and B. J. Nelson, "Modeling and control of untethered biomicrorobots in a fluidic environment using electromagnetic fields," Int. J. Robotics Research, vol. 25, no. 5-6, pp. 527-536, 2006.

[2] J. J. Abbott, O. Ergeneman, M. P. Kummer, A. M. Hirt, and B. J. Nelson, "Modeling Magnetic Torque and Force for Controlled Manipulation of Soft-Magnetic Bodies," IEEE Trans. Robotics, vol. 23, no. 6, pp. 1247-1252, 2007.

[3] (2008) Model eye (8mm pupil) from Gwb International, Ltd. [Online] Available: http://www.gwbinternational.com/model_eye.htm

[4] T. Drummond and R. Cipolla, "Real-Time Visual Tracking of Complex Structures," IEEE Trans. Pattern Analysis and Machine Intelligence, pp. 932-946, 2002.

[5] J. Ens and P. Lawrence, "An investigation of methods for determining depth from focus," IEEE Trans. Pattern Analysis and Machine Intelligence, vol. 15, no. 2, pp. 97-108, 1993.

[6] M. Subbarao and G. Surya, "Depth from defocus: A spatial domain approach," Int. J. Computer Vision, vol. 13, no. 3, pp. 271-294, 1994

[7] R. Luo, X. Y. Yang, X. F. Peng, and Y. F. Sun, "Three-dimensional tracking of fluorescent particles applied to micro-fluidic measurements," J. Micromechanics and Microengineering, vol. 16, no. 8, pp. 1689-1699, 2006.

[8] M. Wu, J. W. Roberts, and M. Buckley, "Three-dimensional fluorescent particle tracking at micron-scale using a single camera," Experiments in Fluids, vol. 38, no. 4, pp. 461-465, 2005.

[9] K. B. Yesin, K. Vollmers, and B. J. Nelson, "Guidance of magnetic intraocular microrobots by active defocused tracking," IEEE/RSJ Int. Conf. Intelligent Robots and Systems, vol. 4, pp. 3309-3314, 2004.

[10] I. Escudero-Sanz and R. Navarro, "Off-axis aberrations of a wideangle schematic eye model," J. Optical Society of America, vol. 16, no. 8, pp. 1881-1891, 1999.

[11] (2008) S5.7010 lens from FCI Ophthalmics. [Online]. Available: http://www.fci-ophthalmics.com/html/retina.html\#lenses

[12] M. P. Snead, M. P. Rubinstein, and P. M. Jacobs, "The optics of fundus examination," Sur. Ophthalmology, vol. 36, no. 6, pp. 439-445, 1992.

[13] G. Smith and D. A. Atchison, The Eye and Visual Optical Instruments. Cambridge University Press, 1997.

[14] D. A. Volk, "Indirect ophthalmoscopy lens for use with split lamp or other biomicroscope," Jan. 6 1998, U.S. Patent 5,706,073.

[15] (2008) Digital Wide Field ${ }^{\circledR}$ lens from Volk Optical Inc. [Online]. Available: http://volk.com/volk-digital-dwf.html

[16] Y. Sun, S. Duthaler, and B. J. Nelson, "Autofocusing in computer microscopy: selecting the optimal focus algorithm," J. Microscopy Research and Technique, vol. 65, no. 3, pp. 139-149, 2004.

[17] E. Hecht, Optics, 4th ed. San Francisco, CA, Addison Wesley, 2002.

[18] Y. Mejia-Barbosa and D. Malacara-Hernandez, "A review of methods for measuring corneal topography," J. Optometry and Vision Science, vol. 78, no. 4, pp. 240-53, 2001.

[19] F. Password, "Phakometric measurement of ocular surface radii of curvature, axial separations and alignment in relaxed and accommodated human eyes," J. Ophthalmic and Physiological Optics, vol. 24, no. 2, pp. $65-73,2004$ 